\title{
A COMPUTER PROGRAM FOR RADIOCARBON AGE CALIBRATION
}

\section{MINZE STUIVER and PAULA J REIMER}

Department of Geological Sciences and Quaternary Research Center

University of Washington, Seattle, Washington 98195

The calibration curves and tables given in this issue of RADIOCARBON form a data base ideally suited for a computerized operation. The program listed below converts a radiocarbon age and its age error os (one standard deviation) into calibrated ages (intercepts with the calibration curve). and ranges of calibrated ages that correspond to the age error. The standard deviation ${ }^{\circ}$ in the calibration curve is taken into account using $0_{\text {total }}=\sqrt{0 s^{2}+{ }^{0} c^{2}}$ (see Stuiver and Pearson, this issue, for details).

the program transforms radiocarbon ages into cal $A D / B C$ (cal BP) ages. Probabilities within the cal age ranges are not included, this feature will be incorporated at later stage. The FORTRAN program and calibration data can be obtained for the cost of materials and shipping (US $\$ 5$, prepaid and payable to the Quaternary Research Center) from the Quaternary Isotope Laboratory on a DS/DD floppy diskette. The calibration data were assembled from this calibration issue, and from the tabulations of Linick, Suess, and and Becker (Radiocarbon, 27, 20-32, 1985).

The current commitment of the Quaternary Isotope Laboratory is to supply the 1986 version of the program. We do not yet pledge continuous updating but will make an attempt if time and budget permit. The program is IBM PC XT compatible; users are responsible for adaptation to non-compatible systems. A visual display (although not given here) is part of the floppy disk version. Future use of the program will surely lead to modifications and we welcome suggestions.

C Radiocarbon Calibration Program CALIB

The program converts radiocarbon ages to calibrated ages as $C$ would be done if one manually plotted the calibration curve data $C$ on an $X-Y$ axis and drew a line through the $Y$-axis corresponding to $C$ the radiocarbon age. Vertical lines drawn through these intercepts $C$ to the $X$-axis, with linear interpolation between data points, give $C$ the cal $A D / B C$ ages $C a l$ BP ages are calculated from 1950 so that $C$ cal $B P=1950$ - Cal $A D$ and $C a 1$ BP $=1949+$ al $B C$. $C$ caifference in converting $B C$ dates is caused by the absence year difference in converting $B C$ dates is caused by the absence of the $C$ zero year in the $A D / B C$ chronology

To convert the standard error in the radiocarbon age into a range C of cal $A D / B C$ (BP) ages the user must first determine whether to use C 1) the laboratory quoted error or 2) increase the quoted error by C known "error multiplier" (Stuiver and Pearson, 1986, Radiocarbon, C 28, 805-838.) With the sample sigma entered, the program calculates $C$ the total sigma for non-marine samples as:

1 Sigma = SQRT((sample sigma)^2 + (calibration curve sigma)^2)

2 Sigma $=\operatorname{SQRT}\left(\left(2^{*} \text { sample sigma }\right)^{\wedge} 2+(\text { calibration curve sigma })^{\wedge} 2\right)$

C (Stuiver, 1982, Radiocarbon, 24, 1-26). The calibration curve sigma C is the average of the standard deviaton of the 2 data points closest C to each intercept of the radid deviaton of the 2 data points closest $C$ the $X$-axis through the intercepts of $Y+1$ Sigma and $Y-1$ Sigma with the calibration curve give the ranges of cal AD/BC ages for 1 Sigma.

$C$ Likewise intercepts of $Y+2$ Sigma and $Y-2$ Sigma give the 2 Sigma

C ranges. For ranges and sample sigmas greater than 100 years the

$C$ ranges are rounded to the nearest ten years. Ranges that overlap or
C are closer together than one year, or ten if rounded, are reported as ne age range.

Marine samples are treated similarly except that the user must determine the Delta $R$ and the uncertainty in Delta $R$ to use for each sample based on its collection location (Stuiver, Pearson, and Braziunas, 1986, Radiocarbon, 28, 2B...) The marine total sigma is taken as:

1 Sigma $=\operatorname{SQRT}\left((\text { sample sigma })^{\wedge} 2+(\text { Delta } \mathrm{R} \text { sigma })^{\wedge} 2\right)$

2 Sigma $=\operatorname{SQRT}\left(\left(2^{*} \text { sample sigma }\right)^{\wedge} 2+(\text { Delta } \mathrm{R} \text { sigma })^{\wedge} 2\right)$.

Three datasets are provided. The twenty year atmospheric record (2) is recommended for most non-marine samples although a ten year record (1) is given for more detailed comparisons of younger samples. The 20 year marine record (3) should be used with all marine samples. *Input from files:

1. $\operatorname{ATM} 10.14 \mathrm{C}$

$10 \mathrm{yr}$ atmospheric record to $2490 \mathrm{cal}$ BC (circa 4200 14-C BP) 2. АТM20.14C

$20 \mathrm{yr}$ atmospheric record to 7210 cal BC (circa 8200 14-C BP)

Format(1. and 2.): Year, Radiocarbon age, Sigma age

3. MARINE. $14 \mathrm{C}$ $(5 \mathrm{X}, \mathrm{F} 9.1,5 \mathrm{X}, \mathrm{I} 5,5 \mathrm{X}, \mathrm{I} 2)$

$20 \mathrm{yr}$ marine model record to $7190 \mathrm{cal} \mathrm{BC}$ (circa 8585 14-C BP) Format: Year, Radiocarbon age $(5 \mathrm{X}, \mathrm{F} 9.1,5 \mathrm{x}, \mathrm{I} 5)$

Output :

1. to printer LPT1 if desired

2. OUTFIL.14C for listing, rename to save

3. PLTFIL.14C for plotting

Format: sample id, \# of intercepts, calibrated ages 1 sigma value, \# of ranges, ranges, 2 sigma value, \# $(1 \mathrm{X}, \mathrm{A} 12, \mathrm{I} 2, \mathrm{n}(\mathrm{F} 10.1,2 \mathrm{X}))$

$2(1 \mathrm{X}, \mathrm{F} 8.1, \mathrm{I} 2, \mathrm{r}(\mathrm{F} 10.1,2 \mathrm{X}))$

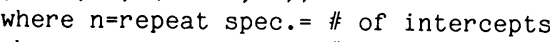
where $r$ repeat spec. $=$ \# of ranges

Subroutines:

INRCP to find the intercept of a radiocarbon age with the calibration curve

Calling sequence: CALL INRCP (V,NPTS, INTPT, NINP) where:

$\mathrm{V}=\mathrm{Y}$ value (Radiocarbon age) to intercept curve NPTS $=$ \# of data points (current dimension $=1000$ ) INTPT $=$ array of intercepting points $(\max =40)$ 
NINP $=\#$ of intercepting points

ABWRT writes calibrated age to unit LO

BPWRT writes age BP to unit LO

RWRT write age ranges to unit LO

Revision date: 7/18/86

C PJ Reimer

Quaternary Isotope Laboratory

Quaternary Research Center AK-60

University of Washington

C Seattle, WA 98195

\$STORAGE: 2

PROGRAM CALIB

COMMON X(1000), Y(1000),S(1000)

COMMON /WRRNG/ RANGE, SIGMAI, NRANG, NPTS, JAD

COMMON /WRINT/ ABINT, BPINT, ENLNE

C

INTEGER*2 AGE, ABINT, BPINT, INTX (40,2)

INTEGER*2 IRANGE $(20,2)$

INTEGER*2 LU( 3$), M \operatorname{MNTX}(40,2), M E N T, N E N T, N \operatorname{INP}, \operatorname{NINTX}(40,2)$

INTEGER*2 RANK $(80)$, SIGAGE, TREF

DEL*4 DELTAR, EINT( 40$)$

REAL*4 INTPT ( 40), MINT (40), RAGE, RANGE $(20,2)$

$\operatorname{REAL} * 4 \operatorname{REFDAT}(4,2), \operatorname{SAMSIG}, \operatorname{SIG} 1, \operatorname{SIG} 2, \operatorname{SIGMA} 1, \operatorname{TEMPR}(40), \operatorname{UNCR}$

CHARACTER COMMA*1, CHSIG*1

CHARACTER CHPM*1, CHSQD*1,FMT*100,IAD $(3) * 2, I C L * 4$

CHARACTER ID*2, IDSAM*12,J JAD*2,LP*1, MREF $(5) * 1$, NAME*10, NAMOUT*10

CHARACTER NAMPLT*10,NREF $(6) * 1, \operatorname{SREF}(6) * 21, \operatorname{SREF} 2(6) * 21, \operatorname{SREF} 1 * 21$

CHARACTER REF $1 * 63, \operatorname{REF} 2(5) * 63, \operatorname{REF} 3(2) * 63, \operatorname{REFAL}(9) * 63$

CHARACTER SREF $3(2) * 21$, STR $1 * 15$, STR $2 * 16$

LOGICAL SKIP, ENLNE

EQUIVALENCE (ID, IDSAM)

C

DATA COMMA/, , /,FMT/' '/

DATA LU $/ 0,200,6 ;$

DATA NAMOUT/'OUTFIL.14C'/,NAMPLT/'PLTFIL.14C'/

DATA NAME/'CAL20.14C\%,IAD/'AD', 'BP', 'AD\%,ICL/'cal '/

C Reference for $10 \mathrm{yr}$ atmospheric record

DATA REF1/'Stulver,M and Becker,B, 1986, Radiocarbon, 28, 2B....'/

DATA SREF1/'(Stuiver and Becker) '/

C References for 20 yr atmospheric record

C

DATA REF2/

\&'Stuiver, M and Pearson, GW, 1986, Radiocarbon, 28, 805-838.'

\&'Pearson, GW and Stuiver, M, 1986, Radiocarbon, 28, 839-632.

\&'Pearson, GW, Pilcher, JR, Baille, MG, Corbett, DM and Qua, F, ',

\&'1986, Radiocarbon, 28, 2B....

\&'Bidecadal weighted average of data from
DATA REFAL/'Linick, TW, Suess, HE and Becker, B, (LSB) 1985, ', \&'Radiocarbon, 27, 20-32. [for the interval 5219-7199 BC ', \&'Stuiver, M, Kromer, B, Becker, B, and Ferguson, CW, (SKBF)' \&'1986, Radiocarbon, 28, 2B....'

\&'Kromer, B, Rhein, M, Bruns, M, Schoh-Fischer, H, Munnich, Ko,', \&'Stuiver, M, and Becker, B, (KRBSMSB) 1986, Radiocarbon, 28,', \&'2B..... [for the interval $5229-7207 \mathrm{BC}$ ]',

'Linick, TW, Long, A, Damon, PE and Ferguson, CW, (LLDF) 1986.', \&'Radiocarbon, $28,2 \mathrm{~B}, \%$

DATA SREF2/'(Stuiver and Pearson)','(Pearson and Stuiver)', \&'(Pearson et al. 1986)', '(20 yr. average of ' $\&$ ' LSB, SKBF, KRBSMSB, ' ' ' and LLDF) '

C

DATA REFDAT/1955.,-500.,-2500,,-5230,,-500.,-2490.,-5210.,-7210./

C Reference for 20 yr marine record

DATA REF3/'Stuiver, M, Pearson, GW, and Braziunas, T, 1986.',

\&'Radiocarbon, 28, 2B.' /

DATA SREF3/'(Stuiver, Pearson and',' Braziunas)

'

DATA STR1/'Calibrated age:'/,STR2/'Calibrated ages: '/

C define character Plus and minus, sigma, and squared and formfeed

CHPM $=\operatorname{CHAR}(241)$
CHSIG $=\operatorname{CHAR}(229)$

CHSIG $=\operatorname{CHAR}(229)$
CHSQD $=\operatorname{CHAR}(253)$

C Open files for text output and plotting

OPEN (6,FILE= 'LPT1')

OPEN $(200$, FILE $=$ NAMOUT,$S T A T U S=$ ' NEW' $)$

$\operatorname{OPEN}\left(300\right.$, FILE $=$ NAMPLT, STATUS $=$ ' ${ }^{N E W}$ ' $)$

DO 20 TORITE 1,3

LO $=$ LU (IWRITE)

LO $=$ LU (IWRITE)
IF (IWRITE.GT.2) THEN

WRITE $(*, 25)$

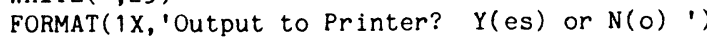

$\operatorname{READ}\left(*,{ }^{\prime}(A)^{\prime}\right)$ LP

IF ( (LP.NE.'Y').AND. (LP.NE.' $\left.\left.y^{\prime}\right)\right)$ THEN

LEND $=2$

GOT

LSE

ENDIF

ENDIF

WRITE(LO, ' $(26 \mathrm{X}, \mathrm{A})$ ') ' 'UNIVERSITY OF WASHINGTON'

WRITE $(L O, '(27 X, A)$ ') 'QUATERNARY ISOTOPE LAB'

WRITE (LO,' $\left.(23 X, A)^{\prime}\right)$ ' 'RADIOCARBON CALIBRATION PROGRAM 1986' WRITE $\left(\mathrm{LO},{ }^{*}\right)$

20 CONTINUE

WRITE $(*, 30)$

FORMAT $\left(/, 1 \mathrm{X},{ }^{\prime}\right.$ Select cal ibration curve dataset.',$/, 1 \mathrm{X}$,

\&'1. $10 \mathrm{yr}$ atmospheric record to $2490 \mathrm{cal}$ BC (circa 4200 14-C BP)',

\&/,1X, 20 yr atmospheric record to $7210 \mathrm{cal}$ BC (circa 8200 14-C BP)', $\& /, 1 x$,

\&'3. 20 yr marine model to 7190 cal BC (circa 8585 14-C BP)') $\operatorname{WRITE}(*, 40)$ 
40 FORMAT(/,1X,'Enter selection: ' )

$\operatorname{READ}(*, *)$ ISET

IF (ISET.GT. 2) THEN

NAME $=$ 'MARINE. $14 \mathrm{C}$ '

LLSEIF (ISET.GT.1) THEN

ELSE

ATM20.14C

NDIF

C Read calibration file

$\operatorname{WRITE}(*, 50)$

50 FORMAT $(/ /, 3 \mathrm{X}$, 'READING CALIBRATION FILE---PLEASE WAIT,$/ /)$ $\operatorname{OPEN}(100, \mathrm{FILE}=$ NAME $)$

$I=1$

100 IF(ISET.LT.3) THEN

$\operatorname{READ}(100,110, E N D=180, E R R=970) \quad Y E A R, A G E, \operatorname{SIGAGE}$

FORMAT ( $5 \mathrm{X}, \mathrm{F} 9.1,5 \mathrm{X}, \mathrm{I} 5,5 \mathrm{X}, \mathrm{I} 2)$

$X(I)=Y E A R$

$\mathrm{Y}(\mathrm{I})=\operatorname{FLOAT}(\mathrm{AGE})$

$S(I)=$ FLOAT (SIGAGE)

ELSE

$\operatorname{READ}(100,120, E N D=180, E R R=970) \quad \mathrm{YEAR}, \operatorname{AGE}$ FORMAT ( $5 X, F 9.1,5 X, I 5)$

$X(I)=Y E A R$

$Y(I)=\operatorname{FLOAT}(A G E)$

ENDIF

NPTS $=I$
$I=I+$

GOTO 100

$180 \quad$ NSAM $=0$

DO $185 \mathrm{~J}=1,4$,

185 CONTINUE

YMAX $=-1 \mathrm{E} 30$

YMAX $=-1$ E 30
DO $190 \mathrm{~J}=1$, NPTS

$\operatorname{YMAX}=\operatorname{AMAX1}(\mathrm{Y}(\mathrm{J}), \mathrm{YMAX})$

190 CONTINUE

200 DO $250 \quad I=1$, LEND

$\mathrm{LO}=\mathrm{LU}(\mathrm{I})$

WRITE $(L 0,205)$ NAME

FORMAT (1X,'Calibration file: ',A10,//)

WRITE $(L 0,210)$

210 FORMAT(' Lab \#',11X,'Radiocarbon', 4X, 'calibrated age(s)', \& $9 \mathrm{X}, \mathrm{\prime}^{\text {References' })}$

WRITE (LO,220)

FORMAT(' ', 18X,'Age BP'

250 CONTINUE

$300 \operatorname{WRITE}\left(*{ }^{*}\right)$

$\operatorname{WRITE}\left(200, '(/ /)^{\prime}\right)$

$\operatorname{WRITE}(*, 310)$

310 FORMAT(' Enter sample ID (or XX to end)' )

$\operatorname{READ}(*, 320)$ IDSAM

FORMAT (A12)

IF((ID.EQ.'XX') .OR. (ID.EQ.' $x x^{\prime}$ )) GOTO 1000

WRITE $(*, 330)$ COMMA

330 FORMAT (' Enter radiocarbon age BP',A,' standard error ')

IF ( (RAGE. LE.0.0).OR. (RAGE.GE. YMAX)) THEN WRITE $(*, 340) 0, \operatorname{INT}\left(\right.$ YMAX $\left.^{-} .5\right)$
340 FORMAT(' VALID RADIOCARBON AGES FOR THIS DATA MUST BE BETWEEN' \&I2,' AND',I5,' YRS BP'

GOTO 305 ENDIF

C For Marine samples, enter reservoir correction Delta $\mathrm{R}$.

C Default Reservoir correction is 400 yrs, Delta $\mathrm{R}=0$. IF (ISET.GT.2) THE

$$
\begin{aligned}
& \text { DELTAR }=0.0 \\
& \text { UNCR }=0.0
\end{aligned}
$$

UNCR $=0.0$

WRITE $(*, 350)$

FORMAT(1X,'Enter reservior correction Delta $\left.\mathrm{R}^{\prime}\right)$

$\operatorname{READ}(*, *)$ DELTAR

WRITE $(*, 360)$

360 FORMAT( $1 \mathrm{X}$,'Enter Delta R standard deviation') $\operatorname{READ}(*, *)$ UNCR

ENDIF

C Print sample ID and age

DO 375 IWRITE $=1$, LEND

LO=LU ( IWRITE)

WRITE $(L 0,370)$ IDSAM, RAGE, CHPM, SAMSIC

FORMAT $(/, 1 \mathrm{X}, \mathrm{A} 12,2 \mathrm{X}, \mathrm{F} 6.1, \mathrm{~A} 3, \mathrm{~F} 5.1,3 \mathrm{X}, \mathrm{I})$

375 CONTINUE

C Subtract reservoir correction and add estimated extension to marine C model

IF(ISET.GT.2) THEN

RAGE = RAGE - DELTAR

NPTS $=$ NPTS +

$X($ NPTS $)=1954$

$Y($ NPTS $)=493$

C Add estimated bomb carbon influence to dataset

NPTS $=$ NPTS +

$X($ NPTS $)=1955$

$Y($ NPTS $)=0$.

$S($ NPTS $)=32$

C Find intercepts of Radiocarbon age with calibration curve

380 CALL INRCP (RAGE, NPTS, INTPT, NINP, INTX)

C Consolidate intercepts that round to the same year

DO $420 \mathrm{~K}=1$, NINP-1

$\operatorname{INK1}=\operatorname{NINT}(\operatorname{INTPT}(K))$

$\operatorname{INK} 2=\operatorname{NINT}(\operatorname{INTPT}(K+1))$

IF (IABS (INK1-INK2).LT.1) THEN

DO $410 \mathrm{~K} 2=\mathrm{K}+1$, NINP- 1

$410 \quad \operatorname{INTPT}(K 2)=\operatorname{INTPT}(K 2+1)$ NINP $=$ NINP-1

ENDIF

420 CONTINUE

C Write to plot file PLTFIL.14C

WRITE(FMT, 430) NINP

430 FORMAT(' (1X,A12,I2, 'I2, ' $\left.(\mathrm{F} 10.1,2 \mathrm{X}))^{\prime}\right)$

WRITE (300,FMT) IDSAM, NINP, (INTPT (K), K=1, NINP) 
C

C Write to OUTFIL.14C for later listing, to screen, and printer

$$
\text { DO } 590 \text { IWRITE }=1 \text {, LEND }
$$$$
\mathrm{LO}=\mathrm{LU}(\text { IWRITE) }
$$

Check calibrated ages for appropriate references. If age falls between datasets give both references.

$\mathrm{KREF}=1$

IF (ISET.GT.2) THEN

$\operatorname{SREF}(1)=\operatorname{SREF} 3(1)$

$\operatorname{SREF}(2)=\operatorname{SREF} 3(2)$

$\mathrm{JREF}=2$

ELSEIF (ISET.GT.1) THEN

TREF $=4$

IREF $=4$

DO $450 \mathrm{~K}=1$, TREF

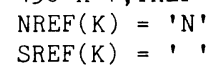

450

$$
\text { CONTINUE }
$$

DO $510 \quad K=1$, NINP

DO $500 \mathrm{~J}=1,4$

$\operatorname{IF}(\operatorname{INTPT}(K) \cdot \operatorname{LT} \cdot \operatorname{REFDAT}(J, 2))$ THEN

$\operatorname{IF}(\operatorname{INTPT}(K) . \operatorname{GT} . \operatorname{REFDAT}(\mathrm{J}+1,1))$ THEN $\operatorname{NREF}(J)=' Y$ '

$\operatorname{NREF}(J+1)=' Y$ '

$\operatorname{MREF}(J)=' Y '$
$\operatorname{MREF}(J+1)=' Y '$ GOTO 510

ELSE$$
\text { NDIF }
$$

$\operatorname{NREF}(J)=' Y$ ' GOTO 510

500 ENDIF CONTINUE

510 CONTINUE

IF(NREF (4).EQ. 'Y') THEN

$\operatorname{NREF}(5)=' Y$ '

$\operatorname{NREF}(6)=' Y$ '

TREF $=6$

ENDIF

JREF $=0$

DO $515 \mathrm{~K}=1$, TREF

IF (NREF (K).EQ.'Y') THEN

$$
\text { JREF }=\text { JREF + } 1
$$

515$$
\text { ENDIF }
$$$$
\text { CONT }
$$$$
\operatorname{SREF}(J R E F)=\operatorname{SREF2}(K)
$$

ELSE

REF(1) = SREF

ENDIF

C

Set label to $A D$ or $B C$

$\operatorname{IF}(\operatorname{INTPT}(1) \cdot \operatorname{LT} \cdot 0.0)$ THEN
$\operatorname{IAD}(1)=' B C \cdot$
$\operatorname{ELSE}$
$\operatorname{IAD}(1)=' A D '$
$\operatorname{ENDIF}$

$\left.\operatorname{WRITE}\left(L O,{ }^{\prime}(A 4, A 2,1 \times \backslash)\right)^{\prime}\right) \operatorname{ICL}, \operatorname{IAD}(1)$

C Print calibrated ages

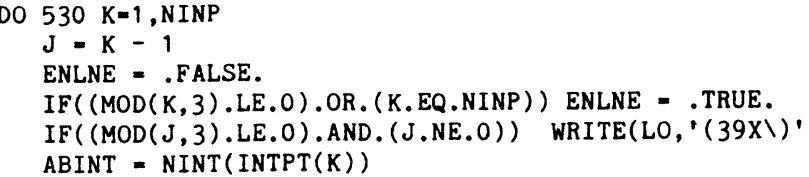

C Check to see if age will print out as zero then change to 1 , since $C$ there is no $O \mathrm{AD} / \mathrm{BC}$

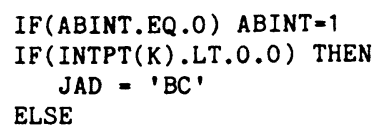

IF (ABINT.EQ.0) ABINT $=1$

IF(INTPT(K).LT.0.0) THEN $J A D={ }^{\prime} B C '$

ELSE

ENDIF

IF(IAD(1) .NE JAD) THEN

WRITE (LO, ' (1X,A4, A2, ' ')') ICL,JAD

ENDIF

CALL ABWRT(LO)

IF (ENLNE) THEN

IF (KREF.LE.JREF) THEN

$\operatorname{IF}(\operatorname{MOD}(K, 3), E Q .2)$ WRITE $\left.(L O, \cdot(6 X \backslash))^{\prime}\right)$

$\operatorname{IF}\left(\operatorname{MOD}(K, 3)\right.$, EQ.1) WRITE $\left(\operatorname{LO},,^{\prime}(12 X \backslash)\right.$ '

$\operatorname{WRITE}\left(L 0,{ }^{\prime}(2 X, A 21)^{\prime}\right)$ SREF(KREF)

LSE WRITE $(L O, *)$

$$
\text { ENDIF }
$$

ENDIF

530 CONTINUE

C calculate ages $B P$

C

IF (ISET.GT.2) THEN

WRITE $(L 0,535)$ DELTAR, CHPM, UNCR, ICL, IAD (2)

535

FORMAT ( $4 X, '$ Delta $R=', F 7.1,1 X, A, F 5.1,4 X, A 4, A 2,1 X 1)$

ELSE

WRITE(LO, ' (32X,A4, A2,1X】)') ICL, IAD(2)

ENDIF

DO $540 \mathrm{~K}=1, \mathrm{NINP}$

$J=K-1$

ENLNE = .FALSE.

$\operatorname{IF}((M O D(K, 3) \cdot L E \cdot 0) \cdot$ OR $(K$, EQ .NINP) ) ENLNE - .TRUE

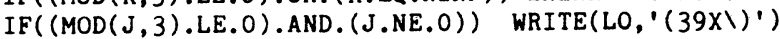

ABINT =NINT (ABS (INTPT (K)) )

$F$ (ABINT.EQ.0) ABINT $=1$

IF(INTPT (K).LT.0.0) TH

BPINT $=1949+$ ABINT

ELSE

BPINT $=1950-A B I N$

ENDIF

CALL BPWRT(LO)

IF (ENLNE) THEN

IF (KREF.LE.JREF) THEN

$\operatorname{IF}(\operatorname{MOD}(K, 3) \cdot \operatorname{EQ} \cdot 2)$
$\operatorname{IF}\left(\operatorname{WOD}(K, 3) \cdot \operatorname{EQ}{ }^{\prime} 1\right)$ 


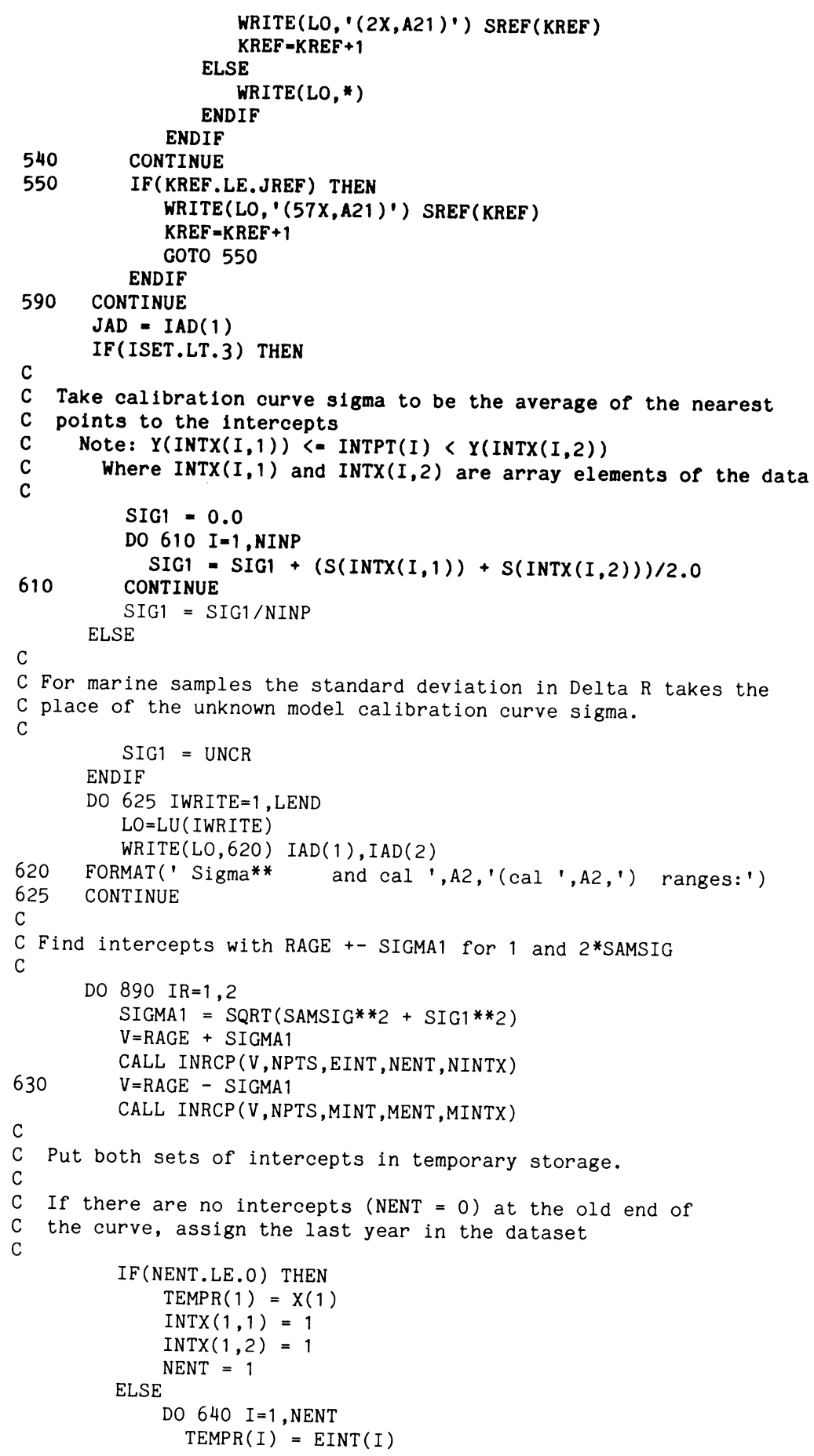

640 ENDIF

$$
\operatorname{INTX}(I, 1)=\operatorname{NINTX}(I, 1)
$$

The modern end of the curve will always have intercepts, though perhaps only with bomb 14C (1955*).

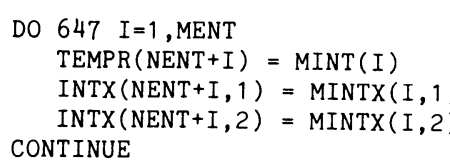


C Assign range values from TEMPR according to RANK

$I C N=I C N+1$

$\operatorname{RANGE}(\operatorname{ICN}, 1)=\operatorname{TEMPR}(\operatorname{RANK}(\mathrm{I}))$

$\operatorname{RANGE}(I C N, 2)=\operatorname{TEMPR}(\operatorname{RANK}(I P 1))$

C If intercept is a turning point in the curve, share it $C$ with the next range, otherwise skip to next

$$
\begin{aligned}
& \text { IF }((Y(I Z) \text {.NE.RS2 }) \text {. AND. }(Y(I Z) \cdot N E \cdot R S 1)) \text { THEN } \\
& \quad \text { SKIP }=\text {.TRUE. }
\end{aligned}
$$$$
\text { ELSE }
$$$$
\text { SKIP }=\text {. FALSE }
$$$$
\text { ENDIF }
$$$$
\text { ELSE }
$$$$
\text { SKIP }=\text {. FALSE. }
$$

690

$$
\text { ENDIF }
$$$$
\text { CONTINUE }
$$$$
\text { NRANG }=\text { ICN }
$$

Consolidate ranges that overlap or have gaps $<10$ year NRANG $=$ 非 of distinct ranges

$$
\begin{aligned}
& \text { ICN }=1 \\
& \text { IF (ICN.GE.NRANG) GOTO } 740 \\
& \text { ICNP1 }=\text { ICN }+1
\end{aligned}
$$

Replace overlapping ranges with maximum and minimum values

$\operatorname{IF}((\operatorname{RANGE}(\operatorname{ICN}, 2)$. GE. RANGE (ICNP1, 1)). OR.

\& ( $\quad \operatorname{RANGE}(\operatorname{ICNP} 1,1)-\operatorname{RANGE}(\operatorname{ICN}, 2))$.LT.10.)) THEN $\operatorname{RANGE}(I C N, 1)=\operatorname{AMIN} 1(\operatorname{RANGE}(\operatorname{ICNP} 1,1), \operatorname{RANGE}(\operatorname{ICN}, 1))$ $\operatorname{RANGE}(I C N, 2)=\operatorname{AMAX1}(\operatorname{RANGE}(I C N P 1,2), \operatorname{RANGE}(I C N, 2))$

C Move rest of ranges into empty slot

DO $735 \mathrm{~K} 2=$ ICNP1, NRANG- 1

$\mathrm{K} 2 \mathrm{P} 1=\mathrm{K} 2+$

$\operatorname{RANGE}(K 2,1)=\operatorname{RANGE}(\mathrm{K} 2 \mathrm{P} 1,1)$

RANGE (K

CONTINUE
NRANG $=$ NRANG -

GOTO 730

ENDIF

$\mathrm{ICN}=\mathrm{ICN}+1$

GOTO 730

740 WRITE(FMT, 741) ICN

WRITE $(300$, FMT $)$ SIGMA1, ICN, $(\operatorname{RANGE}(I, 1)$,

\& RANGE (I, 2), I=1,ICN

741 FORMAT ('(1X,F8.1,I2,'I2,'(F10.1,2X,F10.1))')

C Print ranges

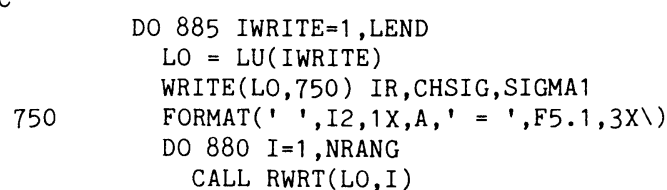

DO 885 IWRITE $=1$, LEND $L O=L U(I W R I T E)$

WRITE (LO,750) IR, CHSIG, SIGM

750 FORMAT (' ', I2, 1X, A, ' = ',F5.1,3X)

DO $880 \quad I=1$, NRANG$$
\text { CALL RWRT(LO, I) }
$$

C Skip to next line after 2 ranges are written
$\operatorname{IF}\left((\operatorname{MOD}(I, 2), E Q, 0)\right.$ AND. (I.NE.NRANG)) $\left.\operatorname{WRITE}\left(L O,{ }^{\prime}(/, 16 \mathrm{X}, 1)\right)^{\prime}\right)$ CONTINUE

WRITE $\left(\right.$ LO $\left.{ }^{*}\right)$

CONTINUE

SAMSIG $=2.0 *$ SAMSIC

890 CONTINUE

C Form feed after 8 samples and write headings again

$\mathrm{NSAM}=\mathrm{NSAM}+$

IF ( (LP.EQ.'Y') .AND. (MOD (NSAM, 6) .EQ.0)) THEN

WRITE $(6,891)$

FORMAT( '1RADIOCARBON CALIBRATION PROGRAM')

WRITE $(6,205)$ NAME

WRITE $(6,210)$

WRITH

ENDIF

GOTO 300

$\operatorname{WRITE}(*$, 975)

FORMAT(' ERROR IN FILE READ')

$1000 \operatorname{WRITE}(*, 1001)$

001 FORMAT $\left(1 \mathrm{X},{ }^{\prime}\right.$ CLOSING FILES'

$\operatorname{CLOSE}(100)$

Form feed to leave room for references if necessary LSAM $=5$

IF(ISET.EQ.2) LSAM $=3$

IF ( (MOD (NSAM, 6) .GE.LSAM) .AND. (LP.EQ.'Y' )) THEN $\operatorname{WRITE}(6,1002)$

1002

ENIF

DO 1200 IWRITE $=1$, LEND

$\mathrm{LO}=\mathrm{LU}($ IWRITE $)$

WRITE $(L O, '(/ / /)$ ')

WRITE $(L 0,1003)$

1003

TFAT (1X, 'Reference

WRITE(LO,1010) REF3(1), REF3(2)

$\mathrm{J}=0$

DO $1005 \mathrm{~K}=1$, IREF

$\mathrm{J}=\mathrm{J}+1$

$\operatorname{IF}(\operatorname{MREF}(K) . E Q . ' Y$ ') THEN

WRITE $(L O, 1010) \operatorname{REF} 2(\mathrm{~J})$

IF (K.EQ.3) THEN

$\mathrm{J}=\mathrm{J}+1$

$\operatorname{WRITE}(L 0,1010) \operatorname{REF} 2(\mathrm{~J})$

ENDIF

IF (K.GT.3) THEN

DO 1004 IRF=1,8

1004

1005 ENDIF WRITE$$
\text { ENDIF }
$$

ELSE

WRITE $(L O, 1010)$ REF

ENDIF

FORMAT $(1 \mathrm{X}, \mathrm{A63})$

WRITE $(L O, 1015)$

FORMAT $(/, 1 \mathrm{X}$, 'Comments: ') 
1020 WRITE $($ LO 0,1020$)$

FORMAT( $1 X^{\prime}, 1955^{*}$ represents influence of bomb C-14')

WRITE $(L 0,1030)$

FORMAT $\left(1 \mathrm{X}, \mathrm{O}^{*}\right.$ represents a "negative" age $\mathrm{BP}$ ')

IF (ISET.LT.3) THEN

WRITE $(L 0,1040)$ CHSQD, CHSQD

1040 FORMAT $\left(1 \mathrm{X}, \mathbf{\prime}^{* *} 1\right.$ sigma = square root of (sample sigma', A, '+ curve sigma', $\left.A,{ }^{\prime}\right) '$ ')

WRITE(LO, 1050) CHSQD CHSOD

1050 FORMAT( $1 \mathrm{X},{ }^{\prime} 2$ sigma $=$ square root of $\left[(2 \text { sample sigma })^{\prime}, \mathrm{A}\right.$, '+ curve sigma', A,']') WRITE $(\operatorname{LO}, 1060)$ IABS(NINT $(X(1)))$

1060 FORMAT( $1 X^{\prime},>^{\prime}$, I5,' BC represents end of calibration data ')

WRITE(LO, 1070) CHSQD, CHSQD

1070 FORMAT $\left(1 X,,^{* *} 1\right.$ sigma $=$ square root of (sample sigma', A,

WRITE $(L O, 1080)$ CHSQD, CHSQD

1080 FORMAT( $1 \mathrm{X},,^{\prime} 2$ sigma $=$ square root of [( 2 sample sigma $)^{\prime}, \mathrm{A}$, + Delta R

090 FORMAT(1X,'>',I5,' BC represents end of calibration data ') ENDIF

1200 CONTINUE

2000 END

\section{SUBROUTINE INRCP $(V, N$, INTPT, NINPT, XYINT)}

C Subroutine to find the intercepts of $\mathrm{V}$ with the straight line

$C$ between two points of a dataset

C V $=\mathrm{Y}$ value for which the intercepts with the function are desired

C INTPT = array of intercepting points

C NINPT $=\#$ of intercepts

$\mathrm{C} N=$ N of data points

C XYINT = element " \#'s of the data array that $\mathrm{V}$ falls between

COMMON X(1000), Y(1000),S(1000)

$\operatorname{REAL} * 4 V, \operatorname{INTPT}(40), M, B, X 3$

INTEGER*2 N,NINPT,XYINT $(40,1)$

NINPT $=0$

10 DO $100 \quad I=2, N$

$\mathrm{X} 1=\mathrm{X}(\mathrm{I}-1)$

$X 2=X(I)$

$\mathrm{Y} 1=\mathrm{Y}(\mathrm{I}-1)$
$\mathrm{Y} 2=\mathrm{Y}(\mathrm{I})$

$\mathrm{Y} 2=\mathrm{Y}(\mathrm{I})$
$\mathrm{IF}(((\mathrm{V} . \mathrm{GE} . \mathrm{Y})$

( (V.GE.Y1).AND. (V.LT.Y2)) .OR. ((V.LE.Y1).AND. (V.GT.Y2))) THEN $M=(Y 2-Y 1) /(X 2-X 1)$

$\mathrm{B}=\mathrm{Y1}-\mathrm{M}^{*} \mathrm{X} 1$

$\mathrm{X} 3=(\mathrm{V}-\mathrm{B}) / \mathrm{M}$

NINPT $=$ NINPT +1

$\operatorname{INTPT}($ NINPT $)=X 3$

$X Y I N T(N I N P T, 1)=I-1$

ENDIF
100 CONTINUE

RETURN

END

SUBROUTINE ABWRT(LO)

COMMON /WRINT/ABINT, BPINT, ENLNE

CHARACTER MARK* ${ }^{*}$, COMMA*1

INTEGER ABINT, BPINT, LO, NBINT

LOGICAL ENLNE

COMMA $=$ ',

MARK $=1 *^{\prime}$

$=\operatorname{IABS}(A B I N T)$

IF (ABINT.LT.1954) THEN

IF (ENLNE) THEN WRITE (LO,' $\left.(1 \mathrm{X}, \mathrm{I} 4 \backslash)^{\prime}\right)$ ) NBINT

ELSE

WRITE(LO, ' ( $\left.1 \mathrm{X}, \mathrm{I} 4, \mathrm{~A} \backslash)^{\prime}\right)$ ) NBINT, COMMA ENDIF

NBINT $=1955$

IF (ENLNE) THEN

WRITE (LO, ' (1X,I4,A $\left.\left.)^{\prime}\right)^{\prime}\right)$ NBINT, MARK

ELSE

RITE (LO, '(1X,I4,2A )') NBINT, MARK, COMMA

RETURN

END

SUBROUTINE BPWRT(LO)

COMMON /WRINT/ ABINT, BPINT, ENLNE

CHARACTER MARK*1, COMMA*

INTEGER ABINT, BPINT, LO

LOGICAL ENLNE

COMMA $=', '$

IF (BPINT.GE.0) THEN

IF (ENLNE) THEN

WRITE(LO,' (1X,I4\)') BPINT

ELSE

WRITE(LO, ' (1X,I4,A ' ' ') BPINT, COMMA

ELSE

BPINT $=0$

IF (ENLNE) THEN

WRITE(LO,' (1X,I4,A $)^{\prime}$ ') BPINT, MARK

ELSE

WRITE (LO, ' $(1 X, I 4,2 A \backslash) ')$ BPINT, MARK, COMMA ENDI

ENDIF

RETURN

END 
SUBROUTINE RWRT(LO,IR)

COMMON X(1000),Y(1000),S(1000)

COMMON /WRRNG/ RANGE, SIGMA1, NRANG, NPTS, JAD

$\operatorname{REAL} * 4 \operatorname{RANGE}(20,2), \operatorname{SIGMA}$

INTEGER NRANG, IRANGE( (2), BRANG(2)

CHARACTER IAD $(3) * 2, I C L * 4, D A S *^{*}, J_{A D * 2}, K A D * 2, R M A R K * 1$

DATA ICL/'cal '/

RANGT = ABS(RANGE $(I R, 2)-\operatorname{RANGE}(I R, 1))$

$I S I G=N I N T(S I G M A 1)$

C Round range values to nearest ten if sigma > 100 and RANGE $>100$ years $C$ Leave out ranges that will round to the same year (or ten years).

IF ( (ISIG. GE. 100). AND. (RANGT.GE.100.)) THEN
IRANGE(1) $=$ NINT (RANGE (IR,1)/10.) $* 10$

$\operatorname{IRANGE}(2)=\operatorname{NINT}(\operatorname{RANGE}(\operatorname{IR}, 2) / 10) *$.

ELSE

$\operatorname{IRANGE}(1)=\operatorname{NINT}(\operatorname{RANGE}(\operatorname{IR}, 1))$

$\operatorname{IRANGE}(2)=\operatorname{NINT}(\operatorname{RANGE}(\operatorname{IR}, 2))$

IF(IABS (IRANGE( (1)-IRANGE(2)).GT.1) THEN

C

Calculate BP ranges

DO $100 \mathrm{~J}=1,2$

$K=2 * J-1$

IF (RANGE (IR,J) .LT.0.0) THEN

$\operatorname{BRANG}(\mathrm{J})=1949-\operatorname{IRANGE}(\mathrm{J})$

$\operatorname{IAD}(K)=' B C '$

ELSE

$\operatorname{BRANG}(\mathrm{J})=1950-\operatorname{IRANGE}(\mathrm{J})$

$\operatorname{IAD}(K)={ }^{\prime} A D '$

100

$$
\text { ENDIF }
$$

Check to see if range is going to print out as zero then change

Co 1 , since there is no $0 \mathrm{AD} / \mathrm{BC}$.

150

DO $180 \mathrm{~J}=1,2$

IF ( IRANGE $(J) \cdot$ EQ.0) THEN

IF (RANGE(IR,J).LT.0.0) THEN $\operatorname{BRANG}(J)=1950$

$\operatorname{IRANGE}(J)=-1$

ELSE

$\operatorname{BRANG}(J)=1949$

$\operatorname{IRANGE}(J)=1$

$$
\text { ENDI }
$$$$
\begin{array}{r}
\text { ENDIF } \\
\text { CONTINUE }
\end{array}
$$

180

1954 AD is last possible year, since after 1954, the bomb C-14 signal overwhelmed the natural variations; therefore any range $>1954$ AD prints $1955^{*}$ and $0^{*} \mathrm{BP}$.
DO $200 \mathrm{~J}=1,2$

IF (RANGE (IR, J).GT.1954.) THEN

$\operatorname{IRANGE}(\mathrm{J})=1955$

RMARK $={ }^{*}$

Check to see if $\operatorname{RANGE}(I R, 1)$ and $\operatorname{RANGE}(I R, 2)$ are either both $A D$

$\mathrm{OR}$ both $\mathrm{BC}$ and the same as the heading printed for calibrated ages.

$\operatorname{IF}((\operatorname{IAD}(1) \cdot \operatorname{EQ} \cdot \operatorname{IAD}(3)) \cdot \operatorname{AND} \cdot(\operatorname{IAD}(1) \cdot E Q \cdot K A D))$ THEN WRITE (LO,' (I I , A Y ') ') IRANGE (J), RMARK

RRITE(LO, ' (A 4, A2, I 4, A ) ' ') ICL, IAD (K), IRANGE (J), RMARK ENDI

$X(1)$ is the first cal year for the dataset. Any range value $>=X(1)$ prints as $>X(1)$ and $>(1949-X(1))$ BP though the actual range is unknown

$$
\begin{aligned}
& \operatorname{ELSEIF}(\operatorname{RANGE}(\operatorname{IR}, J) \cdot \operatorname{LE} \cdot X(1)) \text { THEN } \\
& \operatorname{IRANGE}(J)=\operatorname{NINT}(\operatorname{ABS}(X(1))) \\
& \operatorname{RN} \operatorname{ARK}=1>\text { ' } \\
& \operatorname{IF}((\operatorname{IAD}(1) \cdot \operatorname{EQ} \cdot \operatorname{IAD}(3)) \text {. AND. }(\operatorname{IAD}(1) \cdot \operatorname{EQ} \cdot \operatorname{KAD})) \text { THEN }
\end{aligned}
$$
WRITE (LO, ' (A, I $4 \backslash)^{\prime}$ ') RMARK, IRANGE (J)

ELSE

WRITE(LO, ' (A 4, A2, A, I4\)' ) ICL, IAD(K), RMARK, IRANGE (J) ENDIF

$K A D=I A D(1)$

ELSE

$\operatorname{IRANGE}(J)=\operatorname{IABS}(\operatorname{IRANGE}(\mathrm{J}))$

$\operatorname{IF}((\operatorname{IAD}(1) \cdot \operatorname{EQ} \cdot \operatorname{IAD}(3)) \cdot \operatorname{AND} \cdot(\operatorname{IAD}(1) \cdot \operatorname{EQ} \cdot \operatorname{KAD}))$ THEN WRITE(LO,' (I I \ ')' ) IRANGE (J)

WRITE(LO, ' (A4, A2, I4 \)' ) ICL, IAD (K), IRANGE (J) $K A D=I A D(1)$

$$
\text { ENDIF }
$$

IF(J.LT.2) WRITE(LO,'(A $\left.)^{\prime}\right)$ DASH

200 CONTINUE

C Write BP ranges

C

$\operatorname{LSTBP}=1949-\mathrm{N} \operatorname{INT}(\mathrm{X}(1))$

DO $350 \mathrm{~J}=1$,

IF (J.LT.2) THEN

WRITE ( LO, 310)

FORMAT('(')

ENDIF

IF (BRANG (J).LT.O) THEN

$\operatorname{BRANG}(\mathrm{J})=0$

WRITE (LO, ' (I $4, A \backslash)$ ') BRANG $(J)$, RMARK

ELSEIF (BRANG $(J)$. GE.LSTBP) THEN

$\operatorname{BRANG}(J)=$ LSTBP

RMARK $='>$ '

WRITE(LO,' (A, I $4 \backslash)^{\prime}$ ') RMARK, BRANG (J)

ELSE

WRITE(LO,' (I $\left.4 \backslash)^{\prime}\right)$ ) BRANG (J)

ENDIF 
IF (J.LT.2) THEN

WRITE (LO,' (A\)') DASH

ELSE

WRITE (LO, 320$)$

320

FORMAT ( ' $) ', 1 \mathrm{X} \backslash$

$350 \quad \begin{aligned} & \text { ENDIF } \\ & \text { CONTINUE } \\ & \text { ELSEIF (IRANGE (1).GE.1954) THEN }\end{aligned}$

RMARK $=$ **

$\operatorname{IRANGE}(1)=1955$

WRITE(LO,' (I 4,A $)^{\prime}$ ') IRANGE(1), RMARK

ENDIF

RETURN

END

ERRATUM. Format 1070 should read:

1070 FORMAT $\left(1 \mathrm{X},{ }^{\prime * *} 1\right.$ sigma $=$ square root of (sample sigma', A, \& $\quad$ ' + Delta $R$ sigma', $\left.\left.A,{ }^{\prime}\right)^{\prime}\right)$ 